Proceedings

\title{
Quality of life and psychosocial impact of the lockdown due to the Covid-19 pandemic on patients with cancer: a cross-sec- tional study ${ }^{\dagger}$
}

\author{
Maria Ferrara ${ }^{1}$, Elisa Langiano ${ }^{1}$, Lavinia Falese ${ }^{1 *}$, Antonella De Marco ${ }^{1}$ and Elisabetta De Vito ${ }^{1}$ \\ 1 Department of Human Sciences, Society and Health - Observatory on Health Education- Hygiene Labora- \\ tory - University of Cassino and Southern Lazio \\ * Correspondence: 1.falese@unicas.it
}

Publisher's Note: MDPI stays neutral with regard to jurisdictional claims in published maps and institutional affiliations.

\section{cc) (i)}

Copyright: $(2020$ by the authors. Submitted for possible open access publication under the terms and conditions of the Creative Commons Attribution (CC BY) license (http://creativecommons.org/licenses/by/4.0/).

\begin{abstract}
The aim of the study was to assess the quality of life and the psychosocial impact of the lockdown due to Covid-19 pandemic on cancer patients in Italy using a questionnaire aiming to detect the psychosocial impact of the lockdown. Among the basic needs, psychological and medical support appeared to be prevalent followed by the need of safe transportation to reach the treatment facilities. Internet was the main source of information on the Coronavirus. Although $72.7 \%$ of patients did not give up hospital therapies, 32.6\% complained of variations in the continuity of the treatments. The majority of the sample (73.8\%) was worried of being infected but the $21.9 \%$ didn't share their anxieties and worries with others. The multivariate regression analysis showed that a pessimistic perception of the quality of life was influenced by living in an extra-urban areas) and alone (OR 1.51); while a perception of a reduced physical function resulted affected by the state of anxiety and stress $(\mathrm{OR}=1.8)$ and the difficulties in continuity of medical assistance $(\mathrm{OR}=1.3)$.
\end{abstract}

Keywords: quality of life; Covid-19; pandemic; lockdown; cancer; mental health

\section{Introduction}

Cancer is the second leading cause of death globally and, according to the Italian National Institute of Statistics (ISTAT), it was responsible for an estimated 180,000 deaths among the approximately 600,000 deaths that occurred in 2016 in Italy [1, 2].

In 2019 there were about a thousand new cases of cancer per day in Italy with a prediction for the year 2020 of about 371,000 new cases of malignant tumour. The most common causes of cancer death in 2019 were cancers of breast (53,500 new cases), colorectal $(49,000)$, lung $(42,500)$, prostate $(37,000)$ and bladder $(29,700)$ [2].

In the last years, however, mortality from cancer has been decreasing in both sexes and overall recovery rates have improved, thanks especially to the greater adherence to screening programs, which allow the early detection of the neoplasm and a greater effectiveness of therapies.

As for other chronic degenerative diseases, the onset, course and outcomes of cancer are associated with social factors, lifestyles, but also psychological factors.

Following the Covid-19 outbreak, in February-March 2020, the Italian Government adopted a progressive implementation of measures and limitations until a complete lockdown was reached.

Given the high level of contagiousness, almost every country has adopted restrictive measures of social distancing and home isolation [3]. 
There is no doubt that the Covid-19 disease and the restrictive government measures are harming the mental health of the general population around the world, bringing fear, frustration, anger and a whole series of complex negative emotions [4, 5].

The pandemic represents a traumatic event that has effects at both the individual and population levels. At the individual level, high rates of depression, anxiety, fear, panic, anger and insomnia have been documented in short-term studies conducted primarily in China [6]. At the population level, the pandemic is associated with a number of psychosocial adversities, including economic difficulties and financial losses (due to unemployment and reduced income), school closures, inadequate resources for medical assistance, domestic violence and poor distribution of basic necessities [7]. The fear of contracting the disease and dying, losing their livelihoods and their loved ones, the uncertainty and concerns for the future, social discrimination, and separation from families and caregivers are some of the reason why the pandemic represents a complex and multifaceted source of psychosocial stress $[8,9]$.

The decrees, regulations and protocols that were issued during the pandemic period aimed mostly at the protection of that part of the population considered most vulnerable, especially from the point of view of the physical health, and took less into consideration the psychological impact, which may require specific tailor made interventions.

While most clinical and research efforts have been directed at reducing the effects of the virus on physical health [10], its short and long-term effects on mental health, especially of specific population groups such as the ones living with chronic conditions, have been little investigated [11].

For cancer patients, the severity of the virus and the implemented restrictive measures have been a source of concern and anxiety both due to the fear of increased risk of complications if infected and the fear of no longer receiving the necessary cancer treatment $[12,13]$.

Individuals who had to undergo oncological screening but postponed it for fear of contagion, individuals with delayed treatments because of the emergency, thus with delaying diagnose as well as those patients struggling with a complex pathological situation, they all experienced a strong psychological distress.

In cancer patients, in fact, the increased risk of mortality and the difficulties in accessing health facilities to undergo medical examinations, tests and treatment for fear of becoming infected, is intensifying the risk of developing mental disorders or worsening existing problems [14]. In addition, mass quarantine and expanded restrictions on public transport represent a major barrier to access treatment and support facilities.

When analysing the impact of Covid-19 on cancer patients, it has to be taken into consideration that are individuals may have different personalities and believes and not all of them have faced the emergency at the same stage of their cancer illness.

The patients who were diagnosed with cancer long time before the start of the pandemic, reactivate their fears, feeling of danger, sense of threat of death and a state of alarm that they felt when they discovered their disease.

The patients undergoing chemotherapy or radiotherapy during the pandemic, on the other hand, have already entered a process that help them to regain a sense of control, the so-called adaptation phase. In this phase they are able to handle a good amount of stress and to apply the mechanisms of resilience, i.e. adaptation to the situation.

For those at the beginning of their cancer journey, a diagnosis during the pandemic or right before brings two type of traumas: one related to the discovery of the illness and another one related to the pandemic and its consequences.

Ultimately, patients who were newly diagnosed and admitted for surgery to remove the tumour during the pandemic had a very different experience from what they would have had before the outbreak. The hospital became a building with restricted access in order to protect patients, visitors and medical staff, and this causes a great sense of 
loneliness [15]. The medical team can give a great assistance and care to the patient, but it cannot replace the family.

The aim of this research is to assess the quality of life and the psychosocial impact of the lockdown due to Covid-19 pandemic as well as the perception of changes in the relationship with doctors and caregivers on cancer patients in Italy and to identify possible risks and protective factors for mental health outcomes.

The results of our study can be useful to provide the necessary elements to elaborate socio-assistance interventions aimed at maintaining or improving the quality of life and the taking care of chronicity of cancer patients on the basis of their physical but also psychological needs.

\section{Materials and Methods}

The cross-sectional study was conducted on a specific target group: cancer patients. No aprioristic statistical calculation of sample size was carried out. We collected data after the issue of the Italian Prime Ministerial Decree on 9 March 2020, which extended the measures to contain and fight the spread of the Covid-19 virus throughout the country.

The online survey was carried out in two moments (in April 2020 and then at the start of the second wave in early November 2020) using a Google Form platform and the invitations were launched on social media channels Facebook and Whatsapp. This method makes it possible to promptly collect the perceptions of the chosen target audience, who reported the impressions and feelings while experiencing them.

\subsection{Survey instrument}

We used an online self-administered questionnaire, easy to understand and to fill in, built ad hoc by the Health Education Observatory of the Hygiene Laboratory of the Department of Human Sciences, Society and Health of the University of Cassino and Southern Lazio. The questionnaire consisted of 30 items with standardised answers with the aim of gathering socio-demographic personal information (sex, age, geographical origin, residence, characteristics related to the family environment and lifestyle, working and living conditions, etc.) and clinical information (type of tumour, co-morbidity, type of therapy, etc.).

The perception of quality of life was measured through the health survey Short Form12 (SF-12), validated on the Italian population by Kodraliu et al. (2001) [16]. This instrument helped us to carry out an analysis of the patients' needs through the assessment of general distress level and the presence of symptoms of depression, anxiety and stress related to the coronavirus epidemic.

\subsection{Statistical analysis}

Descriptive statistics were performed to describe the socio-demographic and clinical characteristics of the sample. The respondents' places of residence were recoded using a binary variable (yes/no) named 'Urban area'. This variable was included in the regression model in order to assess the difficulties in reaching health care provider services. We hypothesized that individuals living in the suburbs or small areas would have more difficulty in reaching hospitals or health facilities, especially if the usually use public transportation, compared to those living in urban areas.

In order to assess factors associated with the severity of depressive symptoms, anxiety and stress from the SF-12, multivariate linear regression models were run. Independent variables were: having a pre-existing mental illness disorder, being female. The models were adjusted for different socio-demographic characteristics, such as gender, occupational status, physical and/or psychological comorbidity, subjective perception of health status, family network, level of satisfaction with one's own life, continuity of care. 
Statistical analyses were performed using the EpiInfo 3.5 statistical package, the level of statistical significance was set at $\mathrm{p}<0.05$.

\section{Results}

The sample consisted of 376 participants, mainly female (82.9\%), with an average age of 40.8 years \pm 14.3 SD. Nearly half of the sample (48.1\%) had a high school diploma and $31.6 \%$ had a university degree. Half of the respondents had a stable relationship and lived with their partner $(48.1 \%)$ while few lived alone $(8.6 \%)$. The sample comes from all over Italy and mainly from a suburban area $(74.5 \%)$. Only $18.8 \%$ of the participants was employed and was going to work regularly as before the pandemic. The $17.7 \%$ of the sample was retired, the $14.9 \%$ was on sick leave, the $12.2 \%$ consists in housewives, the $6.6 \%$ was on layoff and the $16.6 \%$ switched to Smart Working during the pandemic. No one reported to be in isolation because they were Covid-19 positive, although the $2.6 \%$ of the sample stated that they were in precautionary isolation.

Some of the respondent had acquaintances/friends/family members who had been infected and were quarantined at home, who were hospitalised or who have died from Covid-19 (respectively 20\%, 10.6\% and 12.2\%). More than $80 \%$ spent more time on the Internet than usual, most frequently for instant messaging $(82.3 \%)$. The Internet results to be the main source of information about the Coronavirus for most of them $(73.9 \%)$ while doctors (specialist or general practitioner) are little mentioned (16\%). Few patients suffered from a pre-existing physical illness (10\%) and a very small percentage $(3.7 \%)$ reported pre-existing psychological distress, most frequently anxiety (39.3\%) and depressive disorders (34.6\%). During the pandemic, almost half of the respondents perceived their health good and fair (respectively $46.5 \%$ and $39.4 \%$ ) while a small percentage (9\%) considered it very poor. The reported primary need was the psychological support $(52.5 \%)$ followed by the medical support (37.3\%).

Using safe transportation to reach hospital facilities for the treatments was also one of the basic needs of the sample (27.8\%). Although $72.7 \%$ of patients did not give up hospital therapies, $32.6 \%$ complained of variations in the continuity of the treatments.

The majority of the sample (73.8\%) was worried of being infected but the $21.9 \%$ didn't share their anxieties and worries with others.

The mean score for the Physical component Summary (PCS) was $64.4 \pm 8.3 S D$, while for the Mental component summary (MCS) it was 63.3 $\pm 9.2 S D$ with lower scores in both the scales belonging to females (PCS males 45.58 \pm 10.54 SD vs. females $40.41 \pm 10.29$ SD; $\mathrm{p}<0.05$; MSC males 47.82 \pm 10.35 SD vs. females $44.60 \pm 10.11 S D ; \mathrm{p}<0.05$ ).

Linear regression models showed a significant correlation between the presence of pre-existing psychological distress and an increase in the severity of depressive-anxiety symptoms (PCS) due to the pandemic $r=0.3 ;(\mathrm{p}<0.05)$.

The multivariate regression analysis showed that a pessimistic perception of the quality of life was influenced by living in an extra-urban areas (OR $=1.23$; IC 95\%: 1.01 2.2; $\mathrm{p}<0.05$ ) and alone (OR 1.51; IC 95\%: 1.1- 2. 3; $\mathrm{p}<0.05$ ); while a perception of a reduced physical function resulted affected by the state of anxiety and stress (OR $=1.8$; IC 95\%: $1.43-3.01 ; \mathrm{p}<0.05)$ and the difficulties in continuity of medical assistance (OR $=1.3 ; \mathrm{IC} 95 \%$ : 1.09- 2.7; $\mathrm{p}<0.05)$.

Furthermore, multivariate regression models adjusted for the period of exposure to the pandemic and related restrictive measures were significantly associated with worse depressive symptoms (OR=1.9; 95\% CI: 1.3-3.2; $\mathrm{p}<0.05)$ and stress (OR=2.1; 95\% CI: 1.73.5; $\mathrm{p}<0.05)$. The risk of severe depressive symptoms, anxiety and stress was higher in females (OR=2.5; 95\% CI: 1.6-3.9; $\mathrm{p}<0.05)$ and in people with pre-existing psychological distress (OR=3.1; 95\% CI: 2.4-4.6; $\mathrm{p}<0.05)$. In addition, we found that subjects who showed higher levels of satisfaction with their lives and with a stable family network reported a 
lowest psychosocial impact of the pandemic (respectively OR=0.4, 95\% CI: 0.1-0.7; $\mathrm{p}<0.05$ and $\mathrm{OR}=0.2,95 \% \mathrm{CI}: 0.06-0.9 ; \mathrm{p}<0.05)$.

\section{Discussion}

Our data confirm that the current pandemic is an unprecedented event in terms of its impact on the mental health of the general population especially those affected by chronic conditions. A second interesting finding of our survey is that levels of anxiety, depression and stress increased over time, being more severe in the last weeks of the pandemic, as confirmed in our regression model adjusted for socio-demographic characteristics of the respondents. This confirms that the duration of the restraint significantly affects not only physical health but also psychological and social health.

In our study, the females were found at higher risk of developing depressive anxiety symptoms, as already shown in a previous study in a small Italian population samples [17] and in previous outbreaks. This result may be due to the higher incidence of anxietydepressive disorders in women [18] and depressive status and mood swings in women [19], also in community samples [20].

Moreover, having a pre-existing mental health problem is a significant risk factor for the development of depressive symptoms, anxiety and stress [5].

This finding suggests the need to provide appropriate and tailored support interventions as early as possible for cancer patients with symptoms of psychological and emotional disorders, a vulnerable segment of the population that were neglected during the initial stages of the pandemic [21, 22].

During the lockdown, participants reported an increase in time spent on the Internet, which was associated with an increased risk of developing mental health problems, thus not confirming our hypothesis of a protective effect played by the Internet on mental health. This result could be due to the diffusion through the Internet of uncontrolled and unreliable information and fake news, which could have increased the levels of anxiety and depressive symptoms in lonely people with lower levels of education [23]. In a situation of health and social risk, communication must be done accurately and there is a need for media professionals to receive adequate training in order to provide impartial and realistic information during catastrophic events.

In our study, being unemployed, retired or housebound was significantly associated with higher levels of anxiety-depressive symptoms. Our results are in line with a study carried out in United Kingdom that showed that, belonging to a socio-economically disadvantaged group, increased gradually the risk of developing problems of psychosocial distress during the first three weeks of the lockdown [24]. It is important, therefore, to undertake comprehensive, multi-level socio-economic initiatives aimed at reducing the negative effect of the pandemic on society.

Finally, good levels of family support were reported by the sample who participated in our survey, this may be due to the fact that the Italian socio-cultural context, with strong family ties and social relationships, may have had a positive impact on the perception of mutual social support [25].

As far as we know, our research is one of the first to be conducted across the Italian peninsula with a fair sample of the Italian cancer population. Validated and reliable assessment tools were used to investigate different domains of health and perception of quality of life level. Therefore, we believe that the higher frequency of depressive anxiety symptoms in our sample could be interpreted as related to COVID-19, although this causal association should be further investigated. We are aware that using an online tool is not the best methodological choice, as it may have excluded older patients or those living in socially disadvantaged settings. However, this choice was necessary in order to reach, in a short time and in a pandemic situation, a specific target of the Italian 
population. Moreover, it must be acknowledged that the data collected are related to depressive or anxious symptoms, which cannot be considered sufficient to formulate a diagnosis of depressive/anxious disorders.

\section{Conclusions}

Although physical isolation and lockdown are essential public health measures to contain the spread of the COVID-19 pandemic, they represent a serious threat to the psychological and social health and well-being of the general population especially those affected by health problems.

The emotional, social and relational difficulties that have emerged require strong resilience. It is important for health professionals, caregiver and social workers to identify the new needs in order to enhance home care interventions, personalise and optimise care, ensure continuity of care and guarantee a high quality of life even in a health emergency situation.

In addressing the measures that can be put in place to deal with the psychosocial impact of the pandemic, a first distinction must be made between interventions during the crisis and interventions after the crisis. The second distinction that needs to be made is between those who have been quarantined because they were infected or have been in contact with infected people, and those who have only undergone lockdown.

The present study has several clinical implications: (a) to promote mass screening campaigns for the general population to identify the presence of sub threshold mental disorders; (b) to disseminate information on how to deal with the mental health consequences of the pandemic; and (c) to support the population at risk - mainly people with pre-existing mental health problems and COVID-19 patients - with tailored innovative psychosocial interventions.

A multi-disciplinary approach involving oncologists, family doctors, social workers, psychologists and, in some cases, psychiatrists, has to be pursued.

During the pandemic the main mental treatment would be directed to counteract fear. Meditation techniques, mindfulness and psychological support as well as online counselling can be very helpful during this time.

After the critical phase, attention will have to be focused on ensuring the well-being at work and monitoring over time [26].

Therefore, the investment in mental health services and programmes at national level, which have suffered from limited funding for years, is now more important than ever.

Author Contributions MF, EL, EDV contributed to the design and coordination of the study. All contributed to the analysis of the questionnaires. ADM was responsible for data collection. MF carried out the statistical analysis. $\mathrm{LF}$ and ADM contributed to the design of the questionnaires and drafting of the manuscript. All authors have read and approved the final manuscript.

Funding: This research received no external funding

Acknowledgments: None

Conflicts of Interest: The authors declare no conflict of interest.

\section{References}

1. WHO. Cancer. Available online: https://www.who.int/health-topics/cancer\#tab=tab_1 (accessed on 2 January 2021)

2. ISTAT. I Numeri del Cancro 2019. Banca-dati AIRTum. Available online: https://www.epicentro.iss.it/tumori/pdf/NC2019operatori-web.pdf (accessed on 2 January 2021)

3. Wasserman, D.; van der Gaag, R.; Wise, J. The term "physical distancing" is recommended rather than "social distancing" during the COVID-19 pandemic for reducing feelings of rejection among people with mental health problems. Eur Psychiatry 2020, 63(1),e52. doi: 10.1192/j.eurpsy.2020.60. 
4. Xiong, J.; Lipsitz, O.; Nasri, F.; Lui, L.M.W.; Gill, H.; Phan, L.; Chen-Li, D.; Iacobucci, M.; Ho, R.; Majeed, A.; McIntyre, R.S. Impact of COVID-19 pandemic on mental health in the general population: A systematic review. J Affect Disord 2020, 277,5564. doi: 10.1016/j.jad.2020.08.001.

5. Hao, F.; Tan, W.; Jiang, L.; Zhang, L.; Zhao, X.; Zou, Y.; Hu, Y.; Luo, X.; Jiang, X.; McIntyre, R.S.; Tran, B.; Sun, J.; Zhang, Z.; Ho, R.; Ho, C.; Tam, W. Do psychiatric patients experience more psychiatric symptoms during COVID-19 pandemic and lockdown? A case-control study with service and research implications for immunopsychiatry. Brain Behav Immun 2020, 87,100-106. doi: 10.1016/j.bbi.2020.04.069.

6. Rubin, G.J.; Wessely, S. Coronavirus: The psychological effects of quarantining a city. BMJ 2020,368,m313. doi: https://doi.org/10.1136/bmj.m313

7. Pfefferbaum, B.; North, C.S. Mental health and the Covid-19 pandemic. N Engl J Medicine 2020,383,510-512. doi: 10.1056/NEJMp2008017.

8. Fiorillo, A.; Gorwood, P. The consequences of the COVID-19 pandemic on mental health and implications for clinical practice. Eur Psychiatry 2020,63,32. doi: 10.1192/j.eurpsy.2020.35

9. Brooks, S.K.; Webster, R.K.; Smith, L.E.; Woodland, L.; Wessely, S.; Greenberg, N.; Rubin, G.J. The psychological impact of quarantine and how to reduce it: rapid review of the evidence. Lancet 2020, 395, 912-920. doi: 10.1016/S0140-6736(20)304608.

10. Onder, G.; Rezza, G.; Brusaferro, S. Case-fatality rate and characteristics of patients dying in relation to COVID-19 in Italy. JAMA 2020, 323, 1775-1776. doi: 10.1001/jama.2020.4683.

11. Kuzman, M.R.; Curkovic, M.; Wasserman, D. Principles of mental health care during the COVID-19 pandemic. Eur Psychiatry 2020, 63, e45. doi: 10.1192/j.eurpsy.2020.54

12. Dinmohamed, A.G.; Visser, O.; Verhoeven, R.H.A; Louwman, M.W.J.; van Nederveen, F.H.; Willems, S.M.; Merkx, M.A.W.; Lemmens, V.E.P.P.; Nagtegaal, I.D.; Siesling, S. Fewer cancer diagnoses during the COVID-19 epidemic in the Netherlands. Lancet Oncol 2020, 21(6), 750-751. doi: 10.1016/s1470-2045(20)30265-5.

13. Liang, W.; Guan, W.; Chen, R.; Wang, W.; Li, J.; Xu, K.; Li, C.; Ai, Q.; Lu, W.; Liang, H.; Li, S.; He, J. Cancer patients in SARSCoV-2 infection: a nationwide analysis in China. Lancet Oncol 2020, 21(3),335-337. doi:10.1016/ S1470-2045(20)30096-6

14. Ueda, M.; Martins, R.; Hendrie, P.C.; McDonnell, T.; Crews, J.R.; Wong, T.L.; McCreery, B.; Jagels, B.; Crane, A.; Byrd, D.R.; Pergam, S.A.; Davidson, N.E.; Liu, C.; Stewart, F.M. Managing Cancer Care During the COVID-19 Pandemic: Agility and Collaboration Toward a Common Goal. J Natl Compr Canc Netw 2020, 20, 1-4. doi: 10.6004/jnccn.2020.7560.

15. Gundavda, M.K.; Gundavda, K.K. Cancer or COVID-19? A Review of Guidelines for Safe Cancer Care in the Wake of the Pandemic. SN Compr Clin Med 2020, 21, 1-11. doi: 10.1007/s42399-020-00632-2.

16. Kodraliu, G.; Mosconi, P.; Groth, N.; Carmosino, G.; Perilli, A.; Gianicolo, E.A.; Rossi, C.; Apolone, G. Subjective health status assessment: evaluation of the Italian version of the SF-12 Health Survey. Results from the MiOS Project. J Epidemiol Biostat 2001, 6, 305-316. doi: 10.1080/135952201317080715.

17. Alonzi, S.; La Torre, A.; Silverstein, M.W. The psychological impact of preexisting mental and physical health conditions during the COVID-19 pandemic. Psychol Trauma 2020, 12,236-238. doi: 10.1037/tra0000840.

18. McLean, C.P.; Asnaani, A.; Litz, B.T.; Hofmann, S.G. Gender differences in anxiety disorders: prevalence, course of illness, comorbidity and burden of illness. J Psychiatr Res 2011,45,1027-1035. doi: 10.1016/j.jpsychires.2011.03.006.

19. Moccia, L.; Janiri, D.; Pepe, M.; Dattoli, L.; Molinaro, M.; De Martin, V.; Chieffo, D.; Janiri, L.; Fiorillo, A.; Sani, G.; Di Nicola, M. Affective temperament, attachment style, and the psychological impact of the COVID-19 outbreak: an early report on the Italian general population. Brain Behav Imm 2020, 87,75-79. doi: 10.1016/j.bbi.2020.04.048.

20. Girolamo, G.; Polidori, G.; Morosini, P.; Scarpino, V.; Reda, V.; Serra, G.; Mazzi, F.; Alonso, J.; Vilagut, G.; Visonà, G.; Falsirollo, F.; Rossi, A.; Warner, R. Prevalence of common mental disorders in Italy: results from the European Study of the Epidemiology of Mental Disorders (ESEMeD). Soc Psychiatry Psychiatr Epidemiol 2006, 41,853-861. doi: 10.1007/s00127006-0097-4.

21. Kaufman, K.; Petkova, E.; Bhui, K.; Schulze, T. A global needs assessment in times of a global crisis: world psychiatry response to the COVID-19 pandemic. BJPsychiatry Open 2020, 6, E48. doi: 10.1192/bjo.2020.25.

22. Jorm, A.F.; Kitchener, B.A.; Reavley, N.J. Mental health first aid training: lessons learned from the global spread of a community education program. World Psychiatry 2019, 18, 142-143. doi: 10.1002/wps.20621.

23. Rovetta, A.; Bhagavathula, A.S. COVID-19-related web search behaviors and infodemic attitudes in Italy: infodemiological study. JMIR Public Health Surveillance 2020,6,9374. doi: 10.2196/19374.

24. Wright, L.; Steptoe, A.; Fancourt, D. Are we all in this together? Longitudinal assessment of cumulative adversities by socioeconomic position in the first 3 weeks of lockdown in the UK. J Epidemiol Community Health 2020,74,683-688. doi: 10.1136/jech-2020-214475.

25. Luciano, M.; Sampogna, G.; Vecchio, V.; Giacco, D.; Mulè, A.; de Rosa, C.; Fiorillo, A.; Maj, M. The family in Italy: cultural changes and implications for treatment. Int Rev Psychiatry 2012,24,149-56. doi: 10.3109/09540261.2012.656306. 
26. Ormel, J.; Cuijpers, P.; Jorm, A.F.; Schoevers, R. Prevention of depression will only succeed when it is structurally embedded and targets big determinants. World Psychiatry 2019,18,111-112. doi: 10.1002/wps.20580. 